\title{
Nonperturbative phase diagram of interacting disordered Majorana nanowires
}

\author{
François Crépin, ${ }^{1}$ G. Zaránd, ${ }^{2}$ and Pascal Simon $^{3}$ \\ 1 Institute for Theoretical Physics and Astrophysics, \\ University of Würzburg, 97074 Würzburg, German \\ 2BME-MTA Exotic Quantum Phases 'Lendület' Group, Institute of Physics, \\ Budapest University of Technology and Economics, H-1521 Budapest, Hungary \\ ${ }^{3}$ Laboratoire de Physique des Solides, CNRS UMR-8502, \\ Université Paris Sud, 91405 Orsay Cedex, France
}

(Dated: August 28, 2018)

\begin{abstract}
We develop a Gaussian variational approach in replica space to investigate the phase diagram of a one-dimensional interacting disordered topological superconducting wire in the strong coupling regime. This method allows for a non-perturbative treatment in the disorder strength, electronelectron interactions and the superconducting pairing amplitude. We find only two stable phases: a topological superconducting phase, and a glassy, non-topological localized phase, characterized by replica symmetry breaking.

PACS numbers: 71.10.pm, 74.45.+c, 74.78.Na, 74.81.-g
\end{abstract}

Introduction. Majorana fermions (MF) have recently attracted a lot of attention in condensed matter systems. They are interesting from a fundamental point of view as emergent exotic quasiparticles [1] but also for their potential applications in quantum computing [2]. In a seminal work, Kitaev [3] constructed a simple model for a one-dimensional (1D) topological superconductor with $p$-wave pairing, hosting Majorana edge states at each end. Parallel to Kitaev's work, a disordered version of the same toy model has been studied by Motrunich et al, who showed that these edge states survive the presence of moderate disorder [9]. Since Kitaev's proposal, many new platforms have been proposed to emulate an effective $p$-wave triplet pairing. Among them, semiconductor wires with spin-orbit and Zeeman interactions in proximity to an $s$-wave superconductor $[4,5]$ have received considerable attention, as materials are now available experimentally. Transport signatures, which may be explained with Majorana zero modes [6-8], were reported in recent experiments.

While small disorder is harmless [9], large disorder in a 1D $p$-wave superconductor with broken time-reversal and spin SU(2) symmetry (class D) can, however, affect the stability of the topological phase and can drive a transition to a non-topological insulating phase $[9,10]$. Impurities can also lead to localized, non-trivial, in-gap states different from Majorana fermions [11], whose transport signatures may, nonetheless, look much alike [12]. Most of the previous studies, however, ignore the effect of electron-electron interactions, which have been shown to modify strongly the domain of stability of the topological phase [13-16]. Therefore, one may wonder whether the topological superconducting (SC) phase survives when both disorder and electron interactions are taken into account or, eventually, some new phase emerges.

The effect of electron-electron interactions on generic disordered 1D $p$-wave superconductors has been ad- dressed recently using Abelian bosonization and the perturbative renormalization group ( $\mathrm{RG}$ ) by Lobos et al. [17]. They concluded that there is a quantum phase transition from a topological superconducting phase to a non-topological localized phase. However, in a large regime of experimental relevance, including the noninteracting and weakly interacting limits, both the effective disorder and the proximity induced gap scale to strong coupling at low energies, a regime outside the reach of perturbative methods.

In this letter, we develop a non-perturbative lowenergy theory for an interacting disordered $p$-wave SC using a Gaussian variational approach (GVA) in replica space [18]. This method has been shown to capture the thermodynamic and transport properties of a disordered wire [19] and even more exotic phases such as the Mott glass, an insulating phase with a non-zero optical conductivity resulting from the competition between a Mott and an Anderson insulating phase [20]. The original approach of Mézard and Parisi, however, cannot be applied to the present problem, and needs be somewhat modified; while superconductivity tends to localize the phase of the Cooper pairs, disorder has a tendency to localize charge carriers in real space and pin their density, the conjugate variable of the superfluid phase. Therefore, unlike previous theories [19,20], both the superfluid phase and its conjugate momentum must be kept in the variational theory and treated at equal footing. With this modification, the GVA is, however, well-suited to capture the competition between disorder and proximity induced superconductivity.

Within the non-perturbative GVA, we find that only two phases are present: a glassy Anderson insulating phase with broken replica symmetry, dominated by disorder, and a replica symmetrical topological SC phase that can support Majorana edge states. We derive the transition line between these two phases. In the non- 
interacting case, we recover using the GVA the transition line previously obtained by Motrunich et al. with the real-space renormalization group apporach which assumes strong disorder [9].

Model. The low energy physics of a semiconductor nanowire with a strong Rashba spin-orbit interaction $\alpha_{R} k_{F}$ ( $k_{F}$ the Fermi momentum), and a Zeeman interaction $E_{Z}$ in proximity to an $s$-wave superconductor (with induced s-wave pairing $\Delta_{S}$ ) can be captured by a model of spinless fermions with $p$-wave pairing $[4,5]$. Treating pairing, electron interactions and disorder on equal footing is a notoriously difficult task. Therefore we follow Ref. [13] and first diagonalize the nanowire Hamiltonian in the presence of spin-orbit and Zeeman interactions, to obtain the dispersion relation $\epsilon_{ \pm}(k)=k^{2} / 2 m \pm \sqrt{\left(\alpha_{R} k\right)^{2}+\left(E_{Z} / 2\right)^{2}}[4,5]$, with $m$ the electrons' effective mass (we set $\hbar=1$ ), with \pm labeling the two bands. Expanding the singlet SC term in this eigenbasis leads to superconducting order parameters of the triplet (within the - and + subbands) as well as of the singlet type (mixing - and + subbands). Majorana edge states require triplet pairing [4, 5, 21-23], which is achieved by tuning the chemical potential $\mu$ to lie within the magnetic field gap, where only the $\epsilon_{-}$subband is occupied, and then turning on a small pairing interaction $[4,5]$. In this regime, however, one can describe the physics of the wire by simply neglecting the empty + band, and by projecting the pairing term, the electron-electron interactions, and the disorder potential to the $\epsilon_{-}$subband.

In the projected theory, the low energy properties are then described using standard Abelian bosonization [24], with the action given as $S=S_{0}+S_{\Delta}+S_{\text {dis }}$ with

$$
\begin{aligned}
S_{0}[\phi, \theta] & =\int_{0}^{\beta} d \tau \int_{0}^{L} d x\left(-\frac{i}{\pi} \partial_{x} \theta(x, \tau) \partial_{\tau} \phi(x, \tau)\right. \\
& \left.+\frac{v}{2 \pi}\left[K\left(\partial_{x} \theta(x, \tau)\right)^{2}+\frac{1}{K}\left(\partial_{x} \phi(x, \tau)\right)^{2}\right]\right) \\
S_{\Delta} & =-\frac{2 \Delta}{\pi a} \int d x d \tau \cos [2 \theta(x, \tau)] \\
S_{\text {dis }} & =-\frac{1}{2 \pi a} \int d x d \tau\left[\xi(x) e^{2 i \phi(x, \tau)}+\text { H.c. }\right]
\end{aligned}
$$

The first term $S_{0}$ describes the physics of the interacting electron fluid in the $\epsilon_{-}$band, with collective plasmon excitations of velocity $v$. The bosonic 'displacement' field $\phi$ (more precisely $\partial_{x} \phi$ ) represents charge density fluctuations, while the phase field $\theta$ is conjugate to it, as expressed by the commutation relation $\left[\phi(x), \theta\left(x^{\prime}\right)\right]=$ $-i \frac{\pi}{2} \operatorname{sign}\left(x-x^{\prime}\right)$. The Luttinger parameter $K$ encodes electron-electron interactions with $K<1$ (resp. $K>1$ ) for repulsive (resp. attractive) interactions. The second term of the action, $S_{\Delta}$ describes triplet pairing. Here $a$ denotes a short length cut-off, and the effective pairing interaction, $\Delta \approx \Delta_{S}\left(\alpha_{R} k_{F}\right) / E_{Z}$, tries to pin the superfluid phase to the minima $\theta=n \pi$. Finally, the last term
$S_{\text {dis }}$ describes backscattering on a Gaussian quenched disorder, $\xi(x)$, satisfying $\overline{\xi(x) \xi^{*}\left(x^{\prime}\right)}=D \delta\left(x-x^{\prime}\right)$. In $S_{\text {dis }}$ we dropped forward scattering terms, since they can be gauged away and do not contribute to localization [24].

The Gaussian variational method. To make progress, we use the replica trick [18]: we introduce $n$ copies of the fields $(\phi, \theta) \rightarrow\left(\phi^{a}, \theta^{a}\right)$ with $a \in[1, n]$, average over the Gaussian disorder, and finally take the limit $n \rightarrow 0$. The replicated action thus obtained reads as

$$
\begin{aligned}
S & =\sum_{a=1}^{n} S_{0}\left[\phi^{a}, \theta^{a}\right]-\frac{2 \Delta}{\pi a} \int \mathrm{d} x \mathrm{~d} \tau \cos \left[2 \theta^{a}(x, \tau)\right] \\
& -\frac{D}{(2 \pi a)^{2}} \sum_{a, b=1}^{n} \int \mathrm{d} x \mathrm{~d} \tau \mathrm{d} \tau^{\prime} \cos 2\left(\phi^{a}(x, \tau)-\phi^{b}\left(x, \tau^{\prime}\right)\right) .
\end{aligned}
$$

As revealed by a simple perturbative RG analysis [17], the RG eigenvalues of the pairing and disorder terms are simply $2-K^{-1}$ and $3-2 K$, respectively, and are both relevant for $1 / 2 \leq K \leq 3 / 2$, where the system flows to strong coupling. Lobos et al. [17] conjectured therefore two phases, separated by a critical line: a topological SC phase dominated by $\Delta$, and a non-topological Andersonlocalized phase, driven by $D$.

To establish the Gaussian variational approach (GVA), we first rewrite the first term of the action Eq. (4) in Fourier space $Q=\left(i \omega_{n}, q\right)$ as $S_{0}[\Psi]=\frac{1}{2 \beta L} \sum_{Q} \Psi^{\dagger}(Q) G_{0}^{-1}(Q) \Psi(Q)$, with $\Psi^{T}=$ $\left(\theta^{1}, \ldots, \theta^{n}, \phi^{1}, \ldots, \phi^{n}\right)$ and

$$
G_{0}^{-1}(Q)=\left(\begin{array}{ll}
{\left[G_{0}^{-1}(Q)\right]_{11}} & {\left[G_{0}^{-1}(Q)\right]_{12}} \\
{\left[G_{0}^{-1}(Q)\right]_{21}} & {\left[G_{0}^{-1}(Q)\right]_{22}}
\end{array}\right)
$$

The $n \times n$ blocks $\left[G_{0}^{-1}(Q)\right]_{i j}, i, j=1,2$, are given by $\left[G_{0}^{-1}(Q)\right]_{11}=v K q^{2} / \pi,\left[G_{0}^{-1}(Q)\right]_{12}=\left[G_{0}^{-1}(Q)\right]_{21}=$ $i q \omega_{n} / \pi$ and $\left[G_{0}^{-1}(Q)\right]_{22}=(v / K) q^{2} / \pi$. The basic idea of the GVA is to approximate the action $S$ of Eq. (4) by the best Gaussian action in replica space, $S \rightarrow S_{G}[\Psi]=$ $\frac{1}{2 \beta L} \sum_{Q} \Psi^{\dagger}(Q) G^{-1}(Q) \Psi(Q)$, with $G^{-1}$ a $2 n \times 2 n$ matrix in replica space. Using the well-known inequality, $F \leq F_{\mathrm{var}}[G] \equiv F_{G}+k_{B} T\left\langle S-S_{G}\right\rangle_{G}$, and minimizing the variational free energy $F_{\text {var }}$ with respect to $G$ we obtain self-consistency equations for $G(Q)$ and, at the same time, an estimate for the free energy $F$. Details of the calculations are presented in the supplemental material [25]. As was shown in Ref. [19], the localized phase of fermions in a disordered potential is described by a replica symmetry breaking (RSB) solution. Therefore, we expect the emergence of a replica symmetry broken solution in the disorder-dominated phase. The superconductivity dominated phase is, however, expected to be replica symmetric (RS). These expectations are indeed confirmed by the detailed analysis presented below. The phase transition between these two phases is, however, found to be of first order, implying that close to the phase boundary both phases are locally stable. Our strategy is 
therefore to find both the RS and RSB solutions, and compare their free energy to determine the stable phase of minimal free energy.

Physical quantities as well as the self-consistency equations involve the so-called connected propagator, $G_{i j}^{c} \equiv$ $\sum_{b} G_{i j}^{a b}$, and its inverse, $\left(G^{-1}\right)_{i j}^{c}=\left(G^{c}\right)_{i j}^{-1} \equiv \sum_{b}\left(G^{-1}\right)_{i j}^{a b}$. The propagator $G^{c}(Q)$ can be shown to be simply the disorder-averaged correlation function of the fields $\theta$ and $\phi$ and, within the GVA, we can express it as

$$
\left(G^{-1}\right)^{c}(Q)=\frac{1}{\pi}\left(\begin{array}{cc}
v K q^{2}+m & i q \omega_{n} \\
i q \omega_{n} & v q^{2} / K+\Sigma\left(\omega_{n}\right)
\end{array}\right) .
$$

A finite value of the renormalized gap, $m$, in this equation sustains superconducting order, while RSB, i.e., a glassy phase manifests itself through the non-analytical structure of the connected self-energy $\Sigma\left(\omega_{n}\right)$.

Superconducting phase: RS self-consistency equations. The replica-symmetrical ansatz always leads to a regular self-energy, $\Sigma\left(\omega_{n}\right)=I\left(\omega_{n}\right)$, and describes a superfluid (or normal) phase. Introducing the variables $\hat{I} \equiv(\pi K / v) I$ and $\hat{m} \equiv \pi m /(v K)$, the self-consistency equations for the renormalized superconducting gap reduce to

$$
\begin{aligned}
m & =\frac{8 \Delta}{\pi a} e^{-2\left[G^{c}\right]_{11}(x=0, \tau=0)} \\
{[G]_{11}^{c}(x=0, \tau=0) } & =\frac{1}{\beta L} \frac{\pi}{v K} \sum_{Q} \frac{q^{2}+\hat{I}\left(\omega_{n}\right)}{D(Q, \hat{I})}
\end{aligned}
$$

with the denominator defined as

$$
D(Q, \hat{I})=\left(q^{2}+\hat{m}\right)\left(q^{2}+\hat{I}\left(\omega_{n}\right)\right)+q^{2} \omega_{n}^{2} / v^{2} .
$$

For $1 / 2 \leq K$, these self-consistency equations always yield a solution with a finite mass $m$, corresponding to the topological superconducting phase. Surprisingly, we have not found any indication for a breakdown of this RS solution in the function $I(\omega)$, and concluded that this solution appears to be locally stable against replica symmetry breaking.

Localized phase: RSB self-consistency equations. The self-consistency equations also admit a stable, one-step replica symmetry-breaking solution (1RSB), similar to the ones found in Refs. [19] and [26]. This solution can be interpreted as a glassy, interacting localized phase. In this phase, the replica structure of the Green's functions and thus the self-consistency equations are more involved [25]. They can be constructed by using the Parisi-parametrization of the replica-matrices, $G_{i j}^{a b}$. In this localized phase, the self-energy is found to develop a singular structure,

$$
\Sigma\left(\omega_{n}\right)=I\left(\omega_{n}\right)+\Sigma\left(1-\delta_{n, 0}\right)
$$

with the RSB appearing through the non-zero value of $\Sigma>0$. The last term in this expression generates a

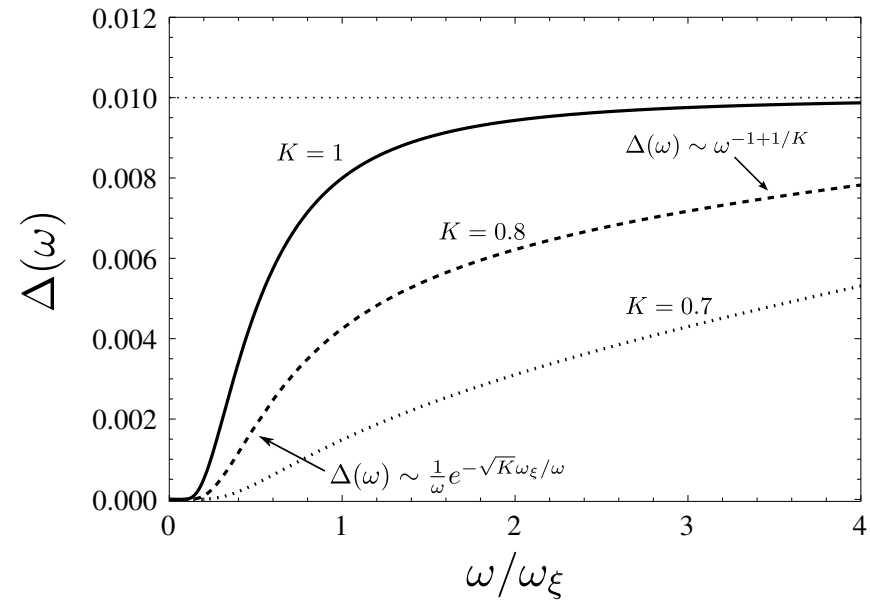

FIG. 1: Effective gap $\Delta(\omega)$ as a function of $\omega / \omega_{\xi}$ for $\Delta_{0} a / v=$ 0.01, $\hat{\Sigma} a^{2}=0.01$ and different values of the Luttinger parameter, $K$. A pseudogap feature emerges below the scale $\omega_{\xi}$.

length scale, $\xi \equiv \hat{\Sigma}^{-1 / 2}=(\Sigma \pi K / v)^{-1 / 2}$, and a corresponding energy scale, $\omega_{\xi} \equiv v / \xi$, which can be identified as the localization length and a corresponding pseudogap, respectively [19].

We analyzed the self-consistency equations within the simplest approximation, $I(\omega) \rightarrow 0$ [19]. In this limit, they reduce to a set of two coupled integral equations for $m$ and $\Sigma$. Surprisingly, however, we found no solutions with $m \neq 0$ and $\Sigma \neq 0$. This result has the important consequence, that the two 'order parameters', $\Sigma$ and $m$ are mutually exclusive within the GVM, i.e., no exotic phase analogous to a Mott glass phase [20] or the BoseFermi glass phase [26] emerges, in agreement with the conjecture of Ref. [17].

To confirm this result, we performed a RG analysis around the $1 \mathrm{RSB}$ fixed point by adding a pairing potential to the Gaussian 1RSB action, $S_{1 \mathrm{RSB}}$

$$
S \equiv S_{1 \mathrm{RSB}}+\sum_{a=1}^{n} \tilde{\Delta} \frac{v}{a^{2}} \int \mathrm{d} x \mathrm{~d} \tau \cos \left[2 \theta^{a}(x, \tau)\right],
$$

and constructing the scaling equation for the dimensionless pairing, $\tilde{\Delta}=\Delta a / v$. The anomalous dimension of $\tilde{\Delta}$ is now scale dependent and, correspondingly, the effective pairing potential at energy $\omega, \Delta(\omega)=\omega \tilde{\Delta}(\omega)$ is found to obey the following scaling equation:

$$
\frac{\mathrm{d} \ln \Delta(\omega)}{\mathrm{d} \ln (\Lambda / \omega)}=1-\frac{\sqrt{\omega_{\xi}^{2}+\omega^{2}}}{K \omega},
$$

with $\Lambda \approx v / a$ a high energy cut-off. At high frequencies $\Delta(\omega)$ therefore behaves as $\Delta(\omega) \sim \omega^{-(1-1 / K)}$, while at small frequencies, $\omega<\omega_{\xi}$, it scales exponentially to zero, $\Delta(\omega) \sim \frac{1}{\omega} e^{-K^{-1 / 2} \omega_{\xi} / \omega}$, consistently with the value of $m=0$ we get from the variational calculation. Therefore, even though $\Delta$ may appear to be a relevant perturbation 


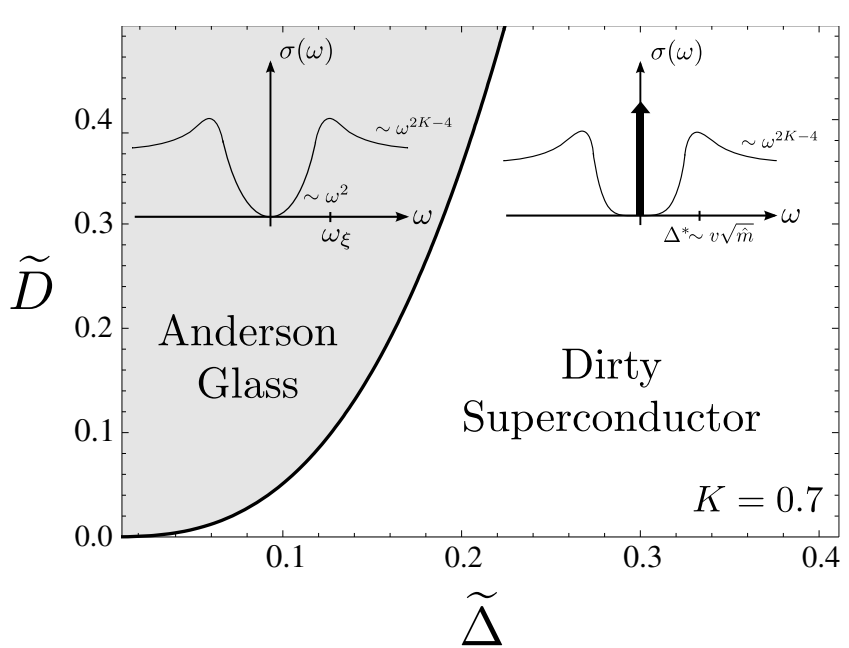

FIG. 2: Phase diagram as a function of the dimensionless disorder parameter, $\widetilde{D}$, and the dimensionless pairing potential, $\widetilde{\Delta}$, for a repulsive interaction $(K=0.7)$, as obtained from the free-energy calculation. A repulsive interaction stabilizes the glassy phase. The two phases are separated by a first-oder line. The insets sketch the optical conductivity $\sigma(\omega)$ in both phases.

at high energies, a finite localization length turns it to be irrelevant at small frequencies, and drives it effectively to zero (see Fig. 1).

We thus conclude from the GVM analysis that - in concordance with the perturbative RG approach of Ref. [17] - only two mutually exclusive phases emerge in the strong coupling regime for $1 / 2 \leq K \leq 3 / 2$ : a glassy phase with a finite localization length, and a topological superconducting phase, separated from the former phase by a first order transition.

Phase diagram. To determine the phase boundary, we computed the variational free energy in both phases. The calculations are detailed in the Supplemental material [25]. In the superconducting phase, we obtain the simple closed expression for the free energy density

$$
\Delta f_{\mathrm{SC}}=f-f_{S C}=\frac{v \hat{m}}{8 \pi}(1-2 K),
$$

while in the glass phase we find

$$
\Delta f_{\mathrm{AG}}=\frac{v \hat{\Sigma}}{8 \pi}(1-3 / K)
$$

Defining the dimensionless disorder as $\widetilde{D} \equiv D a / v^{2}$, and using $\hat{m}=a^{-2}(8 \widetilde{\Delta} / K)^{\frac{K}{2 K-1}}$ and $\hat{\Sigma}=a^{-2}\left(2 \widetilde{D} K^{2}\right)^{\frac{2}{3-2 K}}$ obtained from the self-consistency equations (valid for $\hat{m} \ll a^{-2}$ and $\hat{\Sigma} \ll a^{-2}$ ), and finally comparing $\Delta f_{\mathrm{AG}}$ and $\Delta f_{\mathrm{SC}}$, we obtain the phase diagram in Fig. 2. The phase boundary is given by

$$
\widetilde{D}=\frac{1}{2 K^{2}}\left(\frac{8 \widetilde{\Delta}}{K} \frac{1-2 K}{1-3 / K}\right)^{\frac{3-2 K}{2-1 / K}}
$$

and it changes concavity at $K=1$. For repulsive interactions $(K<1)$, in particular, interactions tend to stabilize the glassy phase, while for attractive interactions they support the SC phase. In the non-interacting case, corresponding to $K=1$, Eq. (13) simplifies to $\frac{\widetilde{\Delta}}{\widetilde{D}}=\frac{1}{2}$. This result is exactly the one derived by Motrunich et al. using a real space renormalization group approach which is suitable in the strong disorder regime. The fact that we recover this result by comparing the variational free energy in both phases from our GVA is highly non-trivial and indicates that the GVA is able to capture the right physics in the strong coupling regime for this system. We presented the phase diagram in Fig. 2 for $K=0.7$, corresponding to repulsive interactions.

Observables. While the topological superconducting phase supports Majorana fermion edge excitations, the localized glassy phase has no single-particle gap and therefore cannot support such protected Majorana edge states. Many proposals have been made to detect signatures of these Majorana edge states by means of transport measurements which are sensitive to edge excitations (see [1]). However, disorder may also lead to localized in-gap states dfferent from Majorana fermions, whose transport signatures may look much alike Majorana edge excitations [10]. In order to distinguish between the two phases depicted in Fig. 2, one may also rely on bulk observables such as the finite frequency optical conductivity $\sigma(\omega)$. For the 1D Anderson glassy phase, such quantity has been computed in Ref.[19] within the GVA and shown to behave as $\sigma(\omega) \sim \omega^{2}$ at low frequency. A power counting argument shows instead that $\sigma(\omega) \sim \omega^{2 K-4}$ at large frequency. We therefore expect a maximum of $\sigma(\omega)$ at the scale $\omega_{\xi}$ corresponding to the pseudo gap energy scale.

In contrast, the topological (dirty) superconducting phase is characterized by a zero-frequency peak in $\sigma(\omega)$ signaling that superconductivity sets in. At large frequency, the optical conductivity is insensitive to superconducting correlations and therefore $\sigma(\omega)$ behaves similarly in both phases. Though we have not been able to obtain a closed form for the low-energy behavior of $\sigma(\omega)$ in the dirty superconducting phase within the GVA, we expect it to exhibit a maximum around the energy scale $\widetilde{\Delta}$. Based on these considerations, we have sketched the expected behavior of the optical conductivity in both phases in Fig. 2.

Conclusions. We developed a Gaussian variational theory in replica space for a 1D topological superconducting wire in presence of electron-electron interaction and disorder. This approach allowed us to capture the competition between pairing and disorder in a non-perturbative way. Two stable phases were found: a topological superconducting phase, and a glassy, non-topological phase with localized carriers, in concordance with the phase diagram conjectured by Lobos et al. [17]. In the glassy phase, p-wave superconductivity was shown to be irrelevant at very small energies. The phase boundary between 
these two phases has been determined analytically.

Acknowledgments. This research has been supported by the French ANR DYMESYS (ANR 2011-IS04-001-01), the Hungarian Research Fund OTKA and the NFÜ under grant Nos. K105149 and CNK80991, respectively.

[1] J. Alicea, Rep. Prog. Phys. 75, 076501 (2012); C. W. J. Beenakker, Annu. Rev. Con. Mat. Phys. 4, 113 (2013); T. D. Stanescu and S. Tewari, J. Phys. Condens. Matter 25, 233201 (2013).

[2] C. Nayak, S. H. Simon, A. Stern, M. Freedman, and S. Das Sarma, Rev. Mod. Phys. 80, 1083 (2008).

[3] A. Yu. Kitaev, Phys. Usp. 44 (suppl.), 131 (2001).

[4] R. M. Lutchyn, J. D. Sau, and S. Das Sarma, Phys. Rev. Lett. 105, 077001 (2010).

[5] Y. Oreg, G. Refael, and F. von Oppen, Phys. Rev. Lett. 105, 177002 (2010).

[6] V. Mourik, K. Zuo, S. M. Frolov, S. R. Plissard, E. P. A. M. Bakkers, and L. P. Kouwenhoven, Science 336, 1003 (2012).

[7] M. T. Deng, C. L. Yu, G. Y. Huang, M. Larsson, P. Caro, and H. Q. Xu, Nano Letters 12, 6414 (2012).

[8] A. Das, Y. Ronen, Y. Most, Y. Oreg, M. Heiblum, and H. Shtrikman, Nat. Phys. 8, 887 (2012).

[9] O. Motrunich, K. Damle, and D. A. Huse, Phys. Rev. B 63, 224204 (2001).

[10] A. C. Potter and P. A. Lee, Phys. Rev. Lett. 105, 227003 (2010); P. W. Brouwer, M. Duckheim, A. Romito, and F. von Oppen, Phys. Rev. B 84, 144526 (2011); P. W. Brouwer, M. Duckheim, A. Romito, and F. von Oppen, Phys. Rev. Lett. 107, 196804 (2011); R. M. Lutchyn, T. D. Stanescu, and S. Das Sarma, Phys. Rev. Lett. 106, 127001 (2011); T. D. Stanescu, R. M. Lutchyn, and S. Das Sarma, Phys. Rev. B 84, 144522 (2011); J. D. Sau, S. Tewari, and S. Das Sarma, Phys. Rev. B 85, 064512 (2012); W. DeGottardi, D. Sen, and S. Vishveshwara, Phys. Rev. Lett. 110, 146404 (2013).

[11] J. Liu, A. C. Potter, K. T Law, P. A Lee, Phys. Rev.
Lett. 109, 267002 (2012); D. Bagrets and A. Altland, Phys. Rev. Lett. 109, 227005 (2012); D. I. Pikulin, J. P. Dahlhaus, M. Wimmer, H. Schomerus, and C. W. J. Beenakker, New J. Phys. 14, 125011 (2012); C-H. Lin, J. D. Sau, and S. Das Sarma, Phys. Rev. B 86, 224511 (2012); D. Rainis, L. Trifunovic, J. Klinovaja, and D. Loss, Phys. Rev. B 87, 024515 (2013); S. Takei, B. M. Fregoso, H.-Y. Hui, A. M. Lobos, S. Das Sarma, Phys. Rev. Lett. 110, 186803 (2013).

[12] E. J. H. Lee, X. Jiang, R. Aguado, G. Katsaros, C. M. Lieber, S. De Franceschi, Phys. Rev. Lett. 109, 186802 (2012). W. Chang, V. E. Manucharyan, T. S. Jespersen, J. Nygard, C. M. Marcus, Phys. Rev. Lett. 110, 217005 (2013).

[13] S. Gangadharaiah, B. Braunecker, P. Simon, and D. Loss, Phys. Rev. Lett. 107, 036801 (2011).

[14] E. M. Stoudenmire, J. Alicea, O. A. Starykh, and M. P.A. Fisher, Phys. Rev. B 84, 014503 (2011).

[15] E. Sela, A. Altland, and A. Rosch, Phys. Rev. B 84085114 (2011).

[16] R. M. Lutchyn, and M. P. A. Fisher, Phys. Rev. B 84, 214528 (2011).

[17] A. M. Lobos, R. M. Lutchyn, and S. Das Sarma, Phys. Rev. Lett. 109, 146403 (2012).

[18] M. Mézard, G. Parisi, J. Phys. 1, 809 (1991).

[19] T. Giamarchi and P. Le Doussal, Phys. Rev. B 53, 15206 (1996).

[20] E. Orignac, T. Giamarchi, and P. Le Doussal, Phys. Rev. Lett. 832378 (1999); T. Giamarchi, P. Le Doussal, E. Orignac, Phys. Rev. B 64245119 (2001).

[21] M. Sato and S. Fujimoto, Phys. Rev. B 79, 094504 (2009).

[22] J. D. Sau, R. Lutchyn, S. Tewari, and S. Das Sarma, Phys. Rev. Lett. 104, 040502 (2010).

[23] J. Alicea, Phys. Rev. B 81, 125318 (2010).

[24] T. Giamarchi. Quantum physics in one dimension, Oxford University Press, Oxford, 2004.

[25] F. Crépin, G. Zarand, P. Simon, supplementary material.

[26] F. Crépin, G. Zarand, P. Simon, Phys. Rev. Lett. 105, 115301 (2010); Phys. Rev. A 85, 023625 (2012). 


\section{Supplementary material for "Majorana fermions in interacting disordered topological superconducting wires"}

\section{DERIVATION OF THE SELF-CONSISTENT VARIATIONAL EQUATIONS}

We summarize here the general formalism of the GVM which led to the set of self-consistent equations both in the superconducting phase (which is replica symmetric) and in the localized phase (described by a 1-step RSB scheme).

In the inverse Green's function $G^{-1}$, we separate the free, Luttinger liquid, part and a self-energy contribution coming from both the pairing and the disorder potentials, such that $G^{-1}=G_{0}^{-1}-\sigma$. Here, $G^{-1}, G_{0}^{-1}$ and $\sigma$ are all $2 n \times 2 n$ matrices, with $n$ the number of replicas. We introduce a double index notation by defining

$$
[G(Q)]_{i j}^{a b}=\left\langle\Psi_{i}^{a}(Q) \Psi_{j}^{b}(-Q)\right\rangle_{G},
$$

where $i, j=1,2$ and $a, b$ run from 1 to $n$, with $\Psi_{1}^{a}=\theta^{a}$ and $\Psi_{2}^{a}=\phi^{a}$. We also use the short-hand notation $Q=\left(i \omega_{n}, q\right) . G^{-1}, G_{0}^{-1}$ and $\sigma$ are therefore $2 \times 2$ block-matrices in the space of $\theta$ and $\phi$, with $n \times n$ blocks (see, e.g. Eq. (5)). The variational free energy has the form

$$
F_{v a r}=-\frac{1}{2 \beta} \sum_{Q} \ln \operatorname{det} G(Q)+\frac{1}{2} \sum_{i, j, a} \sum_{Q}\left[G_{0}^{-1}\right]_{i j}^{a a}(Q) G_{i j}^{a a}(Q)+\frac{1}{2} \sum_{a, b} L \int d \tau\left[V\left[B^{a b}(\tau)\right]-\frac{2 \Delta}{\pi a} e^{-2 G_{11}^{a a}(x=0, \tau=0)}\right],
$$

with

$$
B^{a b}(\tau)=\left\langle\left[\phi^{a}(x, \tau)-\phi^{b}(x, 0)\right]^{2}\right\rangle_{G},
$$

and $V(x)=-2 \frac{D}{(2 \pi a)^{2}} e^{-2 x}$. Differentiation of (S2) with respect to $G$ yields the saddle-point equations. In particular we find that the self-energy matrix $\sigma$ is diagonal in field space, and has the following form in replica space,

$$
\begin{aligned}
\sigma_{22}^{a b}(Q) & = \begin{cases}2 \int_{0}^{\beta} d \tau\left(1-\cos \left[\omega_{n} \tau\right]\right) V^{\prime}\left(B^{a a}(\tau)\right)+2 \int_{0}^{\beta} d \tau \sum_{b \neq a} V^{\prime}\left[B^{a b}\right], \quad(a=b) \\
-2 \beta \delta_{n, 0} V^{\prime}\left(B^{a b}\right) & (a \neq b),\end{cases} \\
\sigma_{11}^{a a}(Q) & =-\frac{8 \Delta}{\pi a} e^{-2\left[G^{c}\right]_{11}(x=0, \tau=0)} \delta_{a b},
\end{aligned}
$$

Note that, in the case of static disorder, off-diagonal quantities, as $B^{a b}$ with $a \neq b$, do not depend on time [19]. This is because off-diagonal elements describe correlations between replicas locked to different minima, but experiencing the same disorder. The experienced random potential being static, these correlations are also time-independent.

The next step is to take the limit $n \rightarrow 0$. We follow Parisi's parameterization of $0 \times 0$ matrices [18]. If $A$ is a matrix in replica space, taking $n$ to 0 it can be parameterized by a couple $(\tilde{a}, a(u))$, with $\tilde{a}$ corresponding to the replica-diagonal elements and $a(u)$ a function of $u \in[0,1]$, parameterizing the off-diagonal elements. To proceed, it is necessary to make an assumption on the form of the self-energy. We actually already know various limits. In the absence of a pairing potential, the localized phase is described by a so-called 1-step replica symmetry breaking (1RSB) solution, for which there exists a value $0<u_{c}<1$ such that $\sigma_{11}\left(u<u_{c}\right)=0$ and $\sigma_{11}\left(u>u_{c}\right)=\sigma$, or equivalently $B\left(u<u_{c}\right)=\infty$ and $B\left(u>u_{c}\right)=B$. In the case where a pairing potential is indeed present we whish to extend this solution by including a mass in the $\theta$ sector. We therefore define $m=-\widetilde{\sigma}_{11}$, which is independent of $Q$. The self-consistent equations take on a simple form, provided we introduce a few auxillary functions that appear naturally in the expression of the so-called connected inverse Green's function

$$
\left[G^{-1}\right]_{i j}^{c} \equiv \sum_{b \neq a}\left[G^{-1}\right]_{i j}^{a b}=\widetilde{G_{i j}^{-1}}-\int_{0}^{1} d u G_{i j}^{-1}(u) .
$$


In the case of a $1 \mathrm{RSB}$ solution we have

$$
\begin{aligned}
& \left(G^{-1}\right)_{11}^{c}=\left(G_{0}^{-1}\right)_{11}+m, \\
& \left(G^{-1}\right)_{22}^{c}=\left(G_{0}^{-1}\right)_{22}+I\left(\omega_{n}\right)+\Sigma\left(1-\delta_{n, 0}\right), \\
& \left(G^{-1}\right)_{12}^{c}=\left(G^{-1}\right)_{21}^{c}=\left(G_{0}^{-1}\right)_{12} .
\end{aligned}
$$

with

$$
\begin{aligned}
& m=\frac{8 \Delta}{\pi a} e^{-2 \widetilde{G_{11}}(x=0, \tau=0)}, \\
& I\left(\omega_{n}\right)=\frac{2 D}{\pi^{2} a^{2}} \int_{0}^{\beta} d \tau\left(1-\cos \left(\omega_{n} \tau\right)\right)\left(e^{-2 \widetilde{B}(\tau)}-e^{-2 B}\right) .
\end{aligned}
$$

The pseudo mass $\Sigma$ is given by $\Sigma=2 \beta u_{c} V^{\prime}[B]$. An extra equation is needed in order to fix the break-point $u_{c}$. It can be obtained by studying the stability of the variational solution $[19,26]$. It turns out that $\phi$ and $\theta$ decouple in the stability analysis and the break-point is the same as in the case of the Bose glass. The final equation for $\Sigma$ reads:

$$
(\Sigma)^{3 / 2}=\frac{2 D}{\pi^{2} a^{2}} \sqrt{\frac{\pi K}{v}} e^{-2 B} .
$$

In order to close the system we also need the following expressions

$$
\begin{aligned}
& \widetilde{G_{11}}(x=0, \tau=0)=\frac{1}{\beta L} \frac{\pi}{v K} \sum_{Q} \frac{q^{2}+\hat{I}\left(\omega_{n}\right)+\hat{\Sigma}}{\left(q^{2}+\hat{m}\right)\left(q^{2}+\hat{I}\left(\omega_{n}\right)+\hat{\Sigma}\right)+q^{2} \omega_{n}^{2} / v^{2}}, \\
& \widetilde{B}(\tau)=\frac{2}{\beta L} \frac{\pi K}{v} \sum_{Q}\left(1-\cos \left(\omega_{n} \tau\right)\right) \frac{q^{2}+\hat{m}}{\left(q^{2}+\hat{m}\right)\left(q^{2}+\hat{I}\left(\omega_{n}\right)+\hat{\Sigma}\right)+q^{2} \omega_{n}^{2} / v^{2}},
\end{aligned}
$$

and

$$
B=\frac{2}{\beta L} \frac{\pi K}{v} \sum_{Q} \frac{q^{2}+\hat{m}}{\left(q^{2}+\hat{m}\right)\left(q^{2}+\hat{I}\left(\omega_{n}\right)+\hat{\Sigma}\right)+q^{2} \omega_{n}^{2} / v^{2}},
$$

where we have introduced $\hat{\Sigma}=(\pi K / v) \Sigma, \hat{I}=(\pi K / v) I$ and $\hat{m}=\pi /(v K) m$. We found no solution of these equations for which both $m$ and $\Sigma$ are non zero.

In the case $\Sigma=0$, the solution becomes replica-symmetric (RS) and the equations simplify to

$$
\begin{aligned}
& \left(G^{-1}\right)_{11}^{c}=\left(G_{0}^{-1}\right)_{11}+m, \\
& \left(G^{-1}\right)_{22}^{c}=\left(G_{0}^{-1}\right)_{22}+I\left(\omega_{n}\right), \\
& \left(G^{-1}\right)_{12}^{c}=\left(G^{-1}\right)_{21}^{c}=\left(G_{0}^{-1}\right)_{12},
\end{aligned}
$$

with

$$
m=\frac{8 \Delta}{\pi a} e^{-2\left[G^{c}\right]_{11}(x=0, \tau=0)},
$$

and

$$
I\left(\omega_{n}\right)=\frac{2 D}{\pi^{2} a^{2}} e^{-2\left[G^{c}\right]_{22}(x=0, \tau=0)} \int_{0}^{\beta} d \tau\left(1-\cos \left(\omega_{n} \tau\right)\right)\left(e^{4\left[G^{c}\right]_{22}(x=0, \tau)}-1\right)
$$

as well as

$$
\left[G^{c}\right]_{11}(x=0, \tau=0)=\frac{1}{\beta L} \frac{\pi}{v K} \sum_{Q} \frac{q^{2}+\hat{I}\left(\omega_{n}\right)}{\left(q^{2}+\hat{m}\right)\left(q^{2}+\hat{I}\left(\omega_{n}\right)\right)+q^{2} \omega_{n}^{2} / v^{2}},
$$

and

$$
\left[G^{c}\right]_{22}(x=0, \tau)=\frac{1}{\beta L} \frac{\pi K}{v} \sum_{Q} e^{i \omega_{n} \tau} \frac{q^{2}+\hat{m}}{\left(q^{2}+\hat{m}\right)\left(q^{2}+\hat{I}\left(\omega_{n}\right)\right)+q^{2} \omega_{n}^{2} / v^{2}}
$$

$G^{c}$ is by definition the inverse of $\left(G^{-1}\right)^{c}$. 


\section{CALCULATION OF THE FREE ENERGY}

\section{Superconducting phase}

The variational free energy is given by:

$$
F=F_{G}+\frac{1}{\beta}\left\langle S-S_{G}\right\rangle_{G}
$$

For convenience, we will compute the free energy per unit volume and replica, that is, $f=F /(n L)$. Using obvious notations we decompose the action as $S=S_{0}+S_{\Delta}+S_{D}$ (see Eq. (4)). With our variational solution in the RS phase, $\left\langle S_{D}\right\rangle_{G}=0$. We are left with three terms to compute:

$$
\begin{aligned}
& f_{1}=F_{G} /(n L), \\
& f_{2}=\frac{1}{n \beta L}\left\langle S_{0}-S_{G}\right\rangle_{G}, \\
& f_{3}=\frac{1}{n \beta L}\left\langle S_{\Delta}\right\rangle_{G} .
\end{aligned}
$$

The most straight-forward term is $f_{3}$. Indeed we have

$$
f_{3}=-n \frac{1}{n \beta L} L \beta \frac{2 \Delta}{\pi a}\left\langle e^{2 i \theta^{a}(x, \tau)}\right\rangle_{G}=-\frac{m}{4},
$$

where we have used Eq. (S10). Not so much complicated is $f_{2}$ :

$$
\begin{aligned}
f_{2} & =-\frac{1}{2 L} \sum_{q, \omega_{n}} \frac{\hat{m}}{\frac{\omega_{n}^{2}}{v^{2}}+q^{2}+\hat{m}} \\
& =-\frac{v \hat{m}}{2 L} \sum_{q>0} \frac{\operatorname{coth}\left(\beta v \sqrt{q^{2}+\hat{m}} / 2\right)}{\sqrt{q^{2}+\hat{m}}} .
\end{aligned}
$$

Finally, we are left with the computation of $f_{1}$. To avoid ambiguities in the definition of the measure in the path integral, we substract $n F_{0}$ to the free energy, with $F_{0}$ the free energy of a Luttinger liquid. We are left to compute $\Delta f_{1}=f_{1}-f_{0}$. We have:

$$
\begin{aligned}
\Delta f_{1} & =-\frac{1}{\beta L} \ln \left[\operatorname{Tr} e^{-S_{G}}\right]+\frac{1}{\beta L} \ln \left[\operatorname{Tr} e^{-S_{0}}\right], \\
& =-\frac{1}{2 \beta L} \sum_{q, \omega_{n}}\left[\ln \operatorname{det} G\left(q, \omega_{n}\right)-\ln \operatorname{det} G_{0}\left(q, \omega_{n}\right)\right] \\
& =-\frac{2}{\beta L} \sum_{q>0} \ln \left[\frac{\sinh (\beta v q / 2)}{\sinh \left(\beta v \sqrt{q^{2}+\hat{m}} / 2\right)}\right] .
\end{aligned}
$$

Finally, we have for, $\Delta f=f-f_{0}$,

$$
\Delta f=-\frac{2}{\beta L} \sum_{q>0} \ln \left[\frac{\sinh (\beta v q / 2)}{\sinh \left(\beta v \sqrt{q^{2}+\hat{m}} / 2\right)}\right]-\frac{v \hat{m}}{2 L} \sum_{q>0} \frac{\operatorname{coth}\left(\beta v \sqrt{q^{2}+\hat{m}} / 2\right)}{\sqrt{q^{2}+\hat{m}}}-\frac{\hat{m}}{4} \frac{v K}{\pi} .
$$

In the limit $\beta \rightarrow \infty, L \rightarrow \infty$, the two first terms conspire to give a finite integral over $q$, and we are left with a particularly simple expression:

$$
\Delta f=\frac{v \hat{m}}{8 \pi}(1-2 K)
$$

$\Delta f<0$ for $K>1 / 2$, which is consistent with the RG. 


\section{Localized phase}

We proceed with a similar calculation in the 1RSB phase. The three pieces to compute are now

$$
\begin{aligned}
\Delta f_{1} & =F_{G} /(n L)-f_{0}, \\
f_{2} & =\frac{1}{n \beta L}\left\langle S_{0}-S_{G}\right\rangle_{G}, \\
f_{3} & =\frac{1}{n \beta L}\left\langle S_{D}\right\rangle_{G} .
\end{aligned}
$$

Again, with start with $f_{3}$ and find

$$
\begin{aligned}
f_{3} & =-\frac{D}{\beta L(2 \pi a)^{2}} L \beta \int_{0}^{\beta} d \tau e^{-2 \widetilde{B}(\tau)}+\left(1-u_{c}\right) \frac{D}{\beta L(2 \pi a)^{2}} L \beta^{2} e^{-2 B}, \\
& =-\frac{D}{(2 \pi a)^{2}} \int_{0}^{\beta} d \tau\left[e^{-2 \widetilde{B}(\tau)}-e^{-2 B}\right]-\frac{\Sigma}{8}
\end{aligned}
$$

where we have used the definition

$$
\Sigma=u_{c} \sigma=u_{c} \frac{2 D}{\pi^{2} a^{2}} e^{-2 B} .
$$

The integral in $f_{3}$ is problematic as it has power-law divergence. It can in principle be cut by the UV cutoff. Since we will neglect $I(\omega)$ everywhere, the consistent approach might be to neglect this integral altogether.

For $f_{2}$ we find:

$$
\begin{aligned}
f_{2} & =-\frac{1}{2 L} \sum_{q, \omega_{n}} \frac{\hat{\Sigma}}{\frac{\omega_{n}^{2}}{v^{2}}+q^{2}+\hat{\Sigma}}+\frac{1}{L} \sum_{q>0} \frac{\hat{\sigma}}{q^{2}+\hat{\Sigma}} \\
& =-\frac{v \hat{\Sigma}}{2 L} \sum_{q>0} \frac{\operatorname{coth}\left(\beta v \sqrt{q^{2}+\hat{\Sigma}} / 2\right)}{\sqrt{q^{2}+\hat{\Sigma}}}++\frac{1}{L} \sum_{q>0} \frac{\hat{\Sigma} / u_{c}}{q^{2}+\hat{\Sigma}} .
\end{aligned}
$$

Finally, in order to compute $\Delta f_{1}$ we use the following formula for the determinant of a 1RSB matrix. If $A=(\tilde{a}, a)$ is such a matrix, with break point $u_{c}$, then

$$
\frac{1}{n} \operatorname{det} A \sim \ln (\tilde{a}-a)+\frac{1}{u_{c}} \ln \frac{\tilde{a}-\left(1-u_{c}\right) a}{\tilde{a}-a} \text { as } n \rightarrow 0 .
$$

Using standard formulas for the determinant of block matrices we find:

$$
\Delta f_{1}=-\frac{2}{\beta L} \sum_{q>0} \ln \left[\frac{\sinh (\beta v q / 2)}{\sinh \left(\beta v \sqrt{q^{2}+\hat{\Sigma}} / 2\right)}\right]-\frac{1}{u_{c} \beta L} \sum_{q>0} \ln \left(1+\hat{\Sigma} / q^{2}\right) .
$$

In the limit $\beta \rightarrow \infty, L \rightarrow \infty$, we find

$$
\Delta f=\frac{v \hat{\Sigma}}{8 \pi}-\frac{\sqrt{\hat{\Sigma}}}{2 \beta u_{c}}+\frac{\sqrt{\hat{\Sigma}}}{4 \beta u_{c}}-\frac{\hat{\Sigma}}{8} \frac{v}{\pi K} .
$$

At this point we can use the marginality condition, which yields,

$$
\hat{\Sigma}=\frac{\pi K}{v} \frac{\sqrt{\hat{\Sigma}}}{u_{c} \beta} .
$$

Collecting all terms, we are left with

$$
\Delta f=\frac{v \hat{\Sigma}}{8 \pi}(1-3 / K)
$$

